Société d'histoire de la révolution de 1848 et des

révolutions du XIXe siècle

Savoirs occultés : du magnétisme à l'hypnose

\title{
Yannick MAREC [dir.], Accueillir ou soigner? L'hôpital et ses alternatives du Moyen Âge à nos jours
}

Mont-Saint-Aignan, Publications des Universités de Rouen et du Havre, 2007, 453 p. ISBN : 978-2-87775-423-1. 35 euros

Claire Fredj

\section{(2) OpenEdition}

Journals

Édition électronique

URL : http://journals.openedition.org/rh19/3898

DOI : $10.4000 /$ rh19.3898

ISSN : $1777-5329$

Éditeur

La Société de 1848

Édition imprimée

Date de publication : 1 juillet 2009

Pagination : 158-160

ISSN : 1265-1354

\section{Référence électronique}

Claire Fredj, « Yannick MAREC [dir.], Accueillir ou soigner? L'hôpital et ses alternatives du Moyen Âge à nos jours », Revue d'histoire du XIXe siècle [En ligne], 38 | 2009, mis en ligne le 04 septembre 2009, consulté le 22 septembre 2020. URL : http://journals.openedition.org/rh19/3898 ; DOI : https:// doi.org/10.4000/rh19.3898

Ce document a été généré automatiquement le 22 septembre 2020.

Tous droits réservés 


\title{
Yannick MAREC [dir.], Accueillir ou soigner? L'hôpital et ses alternatives du Moyen Âge à nos jours
}

\author{
Mont-Saint-Aignan, Publications des Universités de Rouen et du Havre,
} 2007, 453 p. ISBN : 978-2-87775-423-1. 35 euros

\section{Claire Fredj}

1 En septembre 2006, l'hôpital de Fécamp déménage du centre-ville vers le plateau SaintJacques. À l'occasion de ce transfert, l'association Hôpital de Fécamp-Mille ans d'histoire rédige un ouvrage en 2005 et contribue l'année suivante à l'organisation d'un colloque autour du même thème, dont ce livre, réunissant des auteurs nombreux, surtout historiens, est la publication. À partir d'exemples avant tout fécampois, il s'agit d'explorer les rapports entre l'hôpital et la société environnante dans une perspective plus générale. Plusieurs textes sur Paris, Bordeaux, Rouen et Le Havre brisent toutefois la dimension essentiellement locale de l'entreprise. Certains de ces "écarts» géographiques sont d'ailleurs bienvenus dans la comparaison qu'ils suggèrent entre structures hospitalières et sanitaires de villes petites et moyennes.

Une première partie s'intéresse à l'évolution de l'hôpital de Fécamp depuis le $\mathrm{XI}^{\mathrm{e}}$ siècle, époque de sa fondation, jusqu'au XX $\mathrm{XX}^{\mathrm{e}}$ siècle, analysant plus en détail cette histoire au XIX ${ }^{e}$ siècle où le bâtiment, de taille modeste, s'adapte à la croissance urbaine des années 1840-1870. Le nouvel hôpital, financé par un don privé, ouvre en 1849 et reste longtemps un lieu d'assistance pris en charge par un personnel religieux. Son extension au début des années 1880 marque le début de sa transformation en lieu médicalisé. Toujours financé par un don pieux, la maîtrise d'ouvrage est désormais prise en charge par la ville qui s'entoure de professionnels (architectes de la ville, médecins). Mais alors que l'État exige de plus en plus la séparation des fonctions médicales et des fonctions d'accueil, la ville temporise pour des raisons financières. Le jeu des acteurs en charge 
de la politique d'assistance municipale retarde ainsi la mise en place d'un véritable espace de soins, qui n'émerge véritablement que dans l'entre-deux-guerres.

La deuxième partie s'attache au fonctionnement de l'hôpital au XIX ${ }^{e}$ siècle et au début du XXe siècle, par l'étude de la vie quotidienne au sein de l'établissement et par celle des interactions entre personnels religieux, médicaux et administratifs. Frein à la médicalisation ou auxiliaire de celle-ci, la religieuse reste un personnage omniprésent dont on loue le dévouement tout en regrettant un manque de formation médicale qui finit par se développer. C'est aussi l'évolution du monde médical fécampois qui est analysé, avec des figures comme celle de Fauvel, cherchant à créer un service d'hygiène publique sur le modèle du bureau municipal d'hygiène du Havre ou de Léon Dufour, promoteur d'une maternité et d'un service de pédiatrie à l'hôpital. En regard des propositions, réalisations et échecs propres à Fécamp, la lutte contre la tuberculose et la syphilis à Rouen est analysée, particulièrement par le prisme de l'affrontement de clans médicaux et la manière dont la commission administrative des hospices de Rouen profite de ces divergences pour ne pas s'engager dans des voies nouvelles, avant que l'État et le département n'interviennent, stimulant la modernisation et la spécialisation des services hospitaliers.

La troisième partie, enfin, dépasse le cadre de l'hôpital pour s'intéresser à ses «alternatives", éventuellement complémentaires. Un article consacré au service public des secours à domicile à Paris entre 1796 et 1860 montre l'intensité des réflexions menées autour de ce choix permettant d'éviter l'hôpital aux indigents, ce que propose également le dispensaire médicalisé pour enfants malades du Havre, fondé en 1875 par le docteur Gibert, promoteur du premier bureau d'hygiène municipal en France. Moins coûteuse que l'hospitalisation, l'expérience des dispensaires se diffuse en Normandie à la fin du XIX ${ }^{e}$ siècle, notamment à Rouen et à Fécamp. Deux autres articles se penchent sur une innovation fécampoise mise en pratique dès 1894 par le docteur Léon Dufour : « les Gouttes de lait », également créées à Belleville par le docteur Variot. Leur mission est de procurer à l'enfant une nourriture appropriée : des biberons de lait stérilisés y sont distribués, des consultations dispensées, des conseils d'hygiène prodigués. En marge du système hospitalier officiel, cette initiative privée - à Fécamp, elle devient municipale dans les années 1930 - relaie et prolonge l'action de celui-ci sur le terrain, particulièrement auprès des populations défavorisées. Elle contribue également à l'avancement des connaissances médicales sur le rapport entre nutrition et développement de l'enfant. Autre structure complémentaire de l'hôpital à Fécamp, associant établissement public et clinique privée, le sanatorium marin des GrandesDalles, spécialisé dans les tuberculoses osseuse et ganglionnaire. Ouvert en 1922, cet établissement disposant d'un équipement des plus modernes et d'un personnel nombreux, s'inscrit dans la continuité d'une politique globale menée par le département de Seine-Inférieure en faveur de l'enfance en danger.

Accueillir ou soigner, outre son intérêt factuel, bénéficie d'un travail d'édition de qualité, d'illustrations nombreuses et d'annexes souvent intéressantes qui laissent entrevoir la richesse des fonds d'archives et des bibliothèques normandes. L'écriture de l'histoire de la santé profite également de l'ouverture et du classement d'autres fonds, comme ceux du récent service de documentation au siège de la Croix Rouge française ou ceux de l'Instruction publique aux Archives nationales présentés dans le Répertoire méthodique des dossiers relatifs à l'enseignement médical pour le XIX ${ }^{e}$ siècle, établi à partir des séries F 17 et AJ 16. La mémoire des établissements et de la construction des identités 
professionnelles passe également par la recherche ethnographique qui peut accompagner - comme cela a été le cas à Fécamp - le personnel dans son adaptation aux nouvelles structures. Montrant la diversité des financements, des rapports entre autorités, des initiatives locales et individuelles et de leur insertion dans une politique sanitaire à plusieurs échelles mais aussi dans une politique urbaine, cet ouvrage témoigne du développement de l'histoire hospitalière, envisagée à juste titre comme une « histoire-carrefour». 